\title{
Overview and Assessment of Superconducting Technologies for Power Grid Applications
}

\author{
Patrick McGuckin \\ Institute for Energy and the Environment \\ University of Strathclyde \\ Glasgow \\ patrick.mcguckin@strath.ac.uk
}

\author{
Prof. Graeme Burt \\ Institute for Energy and the Environment \\ University of Strathclyde \\ Glasgow \\ graeme.burt@strath.ac.uk
}

\begin{abstract}
It is expected that superconducting technologies will play an important role in the future smart grid because their application brings a host of benefits, most notably a decrease power loss that allows for overload relief, the lowering of voltage levels, power quality enhancement and subsequent grid stability. The phenomenon of superconductivity brings these potential qualities to the grid in the form of a number of technologies analogous to the commonly accepted, conventional types in the form of cabling, fault current limiters, energy storage (ES), generators and transformers. This paper aims to provide an overview of these technologies and their current and potential applications.
\end{abstract}

Keywords- superconductive cabling, superconducting fault current limiter, superconducting magnetic energy storage, generators, transformers, microgrids.

\section{INTRODUCTION}

The UK Energy Research Centre (UKERC) reported that many of the UKs generation and network assets are reaching or have already gone beyond their expected lifetime. The report suggested that the decarbonisation of the electricity system will require significant investment in the replacing of primary assets and as a result, there appears to be potential in the coming years to replace aging equipment with new, innovative and higherperforming technologies in the strive towards a 'cleaner' and 'smarter' power grid [1]. Interest in the phenomenon of superconductivity and its potential applications in the power sector is growing and has the potential to facilitate the required grid modernization through increasing efficiency and capacity whilst reducing losses and $\mathrm{CO}_{2}$ emissions, as well as improving energy security [2]. There are three inherent characteristics that define a superconductor: [3]

- Extremely high current density allows smaller and lighter devices

- Zero resistivity below a critical temperature, lowers the losses allowing higher efficiency

- An abrupt phase change from superconducting to the 'normal' state can be used to produce dramatic changes in impedance in a fraction of a second.
The European Network of Transmission System Operators for Electricity (ENTSO-E) included superconductors as part of their roadmap for 2017-26 under the "Power System Modernisation" and "Security and Stability" visions. Through the advancement of "New Materials and Technologies", the aim is to maintain robust and cost effective infrastructure and more specifically, the development of Superconducting Fault Current Limiters (SFCLs) to avoid strong short circuit currents in new grid architectures. The timescale for this is set at 201722. Furthermore, the aim is to assess the potential contribution to grid controllability via frequency and voltage stability, power quality and creation of synthetic inertia through new large scale power technologies such as High Voltage Direct Current (HVDC), Voltage Source Converters (VSC), Superconductivity (SFCL and cables) and energy storage, with a later timescale set of 2020-26 [4]. With superconductivity forming a part of ENTSO-E's vision for the next 8 years it is evident that superconductive technology is going to play a part in transmission system operators' (TSO) research and development plans across Europe, providing a key boost in advancing the maturity of such technologies.

This paper is intended to provide an overview of the superconductive technologies applicable to the power sector, their specific applications at the forefront of today's research and the challenges preventing widespread adoption and commercialisation. The current and expected technological and market maturity are also suggested.

\section{SuPERCONDUCTING TECHNOLOGIES}

There are predominantly 5 technologies at the forefront of research in the energy sector based on superconductive materials: cabling (AC and DC), Superconducting Fault Current Limiters (SFCL), Superconducting Magnetic Energy Storage (SMES), transformers and rotating machinery. This section will consider each in turn.

\section{A. Cabling}

Superconductive cabling, be it AC or DC, provides certain advantages over conventional options such as negligible transmission losses and significantly enhanced transmission capacity, in turn permitting increased power transmission at lower voltages - this can eradicate the need for new space and 
costly transformers. The compact nature of a superconductive cable and the subsequent reduced right of way requirements also allows for space conservation on the cable route while the lack of stray heat and magnetic field emissions permits installation immediately adjacent to existing cabling in underground conduits, without interference. These benefits can open the door for higher capacity transmission corridors from crucial generation sites be it on or off shore and the future potential for increased interconnectivity.

Superconductive cabling has achieved the most in-field operational experience worldwide, with projects successfully completed or planned in Europe, USA and Asia in particular [5]. The AmpaCity project in Germany for instance eradicated the need for a large $110 / 10 \mathrm{kV}$ substation by carrying the equivalent power at $10 \mathrm{kV}$ requiring only medium voltage switchgear and thus saving valuable space [6]. Korea and Japan are making advances through in-grid demonstration projects on Jeju Island [7] and in a substation in Yokohama [8] respectively. There is also a project in advanced stages to integrate a superconducting feeder cable into the city railway network [8].

Superconducting AC transmission however still exhibits power loss despite possessing zero impedance through 3 main channels: hysteresis loss through a change in the magnetic field, eddy current loss through the cable's metal support tube or through the metal matrix of the high temperature superconducting (HTS) tapes, and coupling loss from the interaction of adjacent HTS tapes- twisting tapes around one another can compensate for this. However, by far the largest contributor to AC loss comes through hysteresis where, as the ratio of AC current to critical current increases, the power loss increases exponentially [9].

Despite AC transmission being the norm for so long, recent advancements in power electronic devices and control systems along with the uptake in renewable energy sources (RES), the trend towards HVDC systems and their coexistence with AC networks is on the rise. More than 180 HVDC projects are reportedly operational across the globe [10].

The trend toward long distance HVDC presents an opportunity for superconductive cables to make a significant advancement in the transmission sector. In Europe, the vision is being explored through European Commission funded BEST PATHS - DEMO5 project with the aim of realizing a $3.2 \mathrm{GW} / 10 \mathrm{kA} / 320 \mathrm{kV}$ HVDC cable manufactured from medium temperature superconducting material, MgB2. Although possessing a lower critical temperature than standard HTS, it has significantly lower raw material costs. Testing is expected to commence in 2018 [11] with the aim of contributing to a panEuropean multi-terminal HVDC power transmission network.

Overall, DC superconductive cabling presents a very attractive alternative to AC equivalents as well as conventional HVDC and utilises the advantages of superconductivity most effectively. With no reactive power, insulation or AC loss to be concerned with, the compactness and less complex design of the DC cable render it likely to receive continued development for integration into future DC transmission networks and links, providing higher capacity at voltages lower than those conventionally adopted.

\section{B. Fault Current Limiters}

SFCLs make use of the abrupt phase change that a superconductor undergoes when its critical current is exceeded, reverting to a state of relatively high resistance. An SFCL limits the fault current and crucially, greatly reduces the amplitude of fault current in the first quarter cycle significantly better and faster than other forms of current limiters. SFCLs thus allow for the paralleling of nearby urban networks, providing increased interconnectivity and new means of managing and re-routing power, protection of RES and the integration of microgrids.

There are various topologies of SFCL: resistive, inductive and hybrid. The resistive type is compact and lightweight whilst rapid and automatic at fault current suppression. The inductive type offers faster recovery without the requirement of a cryogenic-room temperature interface since the superconducting element is coupled inductively. While the hybrid type employs either both resistive and inductive or one or the other in tandem with a fast acting switch. That can allows the bypassing of the superconducting element before quenching enhancing response and subsequent recovery times [12].

There are also competing non-superconducting technologies available for fault current limitation. These include solid-state limiters (SSFCL) [13], standalone fuses [13], and limiting reactors [14]. SSFCLs can offer a high level of control and almost instant recovery times but lack in maturity while standalone fuses are well-established and inexpensive but limited to single use requiring replacement by personnel. Limiting reactors are another well-established option but possess resistance $(25 \mathrm{~kW}$ loss $)$ during normal operation.

At present, the majority of SFCLs under field test are resistive SFCLs or inductive saturated iron-core SFCLs [12]. An in-grid implementation of a resistive 3.4MVA SFCL was conducted in Milan, Italy from 2012-14 with capacity upgraded in 2016 to $15.4 \mathrm{MVA}$, which successfully demonstrated the limitation of a 3-phase short circuit current [15]. Successful implementations have also been seen in the UK where the permanent installation of two SFCLs in Birmingham as part of a $£ 17 \mathrm{~m}$ project allowed the connection of adjacent networks through new bus bar ties [16]. A German collaborative project ASSIST also developed and implemented a resistive SFCL that allowed the connection of an industrial company to the grid and is estimated to save around $36 \mathrm{MWh}$ per year when compared to the use of a conventional limiting reactor [17].

SFCL have also demonstrated improvement in the fault ride through capabilities of integrated microgrids and their connected RES. A reported comparison between a conventional low-voltage ride through (LVRT) protection scheme - a dynamic voltage restorer DVR) - and a superconducting based scheme for RES-rich microgrids claimed an economic advantage for the SFCL and an added advantage of permitting integration of energy storage for improved power stabilization control through a fault [18]. The integration of a SFCL in conjunction with a SMES offers improved fault ride through for microgrids as well as reducing grid-connected fault levels to the 
level of islanded mode, eradicating the need for alternative protection settings [19].

In relation to DC application, SFCL offers tandem operation with DC circuit breakers that quickly restrains fault current such as to reduce the required interrupting capacity [20], enhancing their dynamic thermal stability and reliability while also reducing voltage sag [21]. Various types of SFCLs have been proposed for operation in HVDC systems, with a successful patent application achieved for a hybrid-type superconducting DC circuit breaker using arcing induction evidence that the area harbours interest and potential [22]. The resistive SFCL is reported as the best choice for HVDC systems [23] while it is concluded in [24] after a comparison that the saturated iron-core SFCL is the most likely candidate for commercialisation and application in future DC grids due to its fast recovery and low energy characteristics. Clearly it is still open to further study and demonstration as to which is the more suitable technology, but it likely will come down to the specific application of the device. The need for DC protection technologies to support the HVDC "Supergrid" vision could provide a pathway for the integration of SFCLs into the DC transmission sector because a commercially available and accepted high voltage DC circuit breaker capable of operating sufficiently in a multi-terminal system has yet to be realised. SFCLs are reported to be the most attractive of all available superconducting devices with market maturity anticipated by 2025 - earlier than any other superconducting technology [5].

\section{Magnetic Energy Storage}

Superconducting Magnetic Energy Storage (SMES) comprises inducing a DC current in a coil constructed of superconducting material submerged in liquid helium that subsequently generates a dynamic magnetic field. Whilst in the superconducting state, current can circulate with almost negligible losses, achieving efficiencies of $>95 \%$ [25]. The inherent characteristic qualities are a rapid charge/discharge response of a few milliseconds whilst also providing a very fast full discharge time, high power density as well as the capability of a very high number of cycles, allowing a longer service lifetime. Such qualities render suitability of SMES systems for use in addressing voltage, frequency or power quality issues in applications such as microgrid control [26]. Similar forms of ES competing with SMES are supercapacitors [27] and flywheels [28]. Possessing similar properties to SMES, supercapacitors are suited to high-power, short-duration applications however possess weaker efficiencies (70-85\%) and higher self-daily discharge (20-40\%) [27] while flywheels exhibit high efficiencies of $>90 \%$ and service lifetimes of $>20$ years with fast recharge and response but again possess high levels of daily self-discharge in comparison to SMES [28].

Live, successful in-grid demonstrations of operational SMES systems are few and far between however there is a 3year project active in Italy, "DRYGRID4SMES", which involves research and development of a prototype cryogen-free SMES system based upon the $\mathrm{MgB}_{2}$ and could demonstrate a new method of cooling via heat exchanger as opposed to more common liquid at cryogenic temperatures [29]. The development of a Hybrid Energy Storage System (HESS)

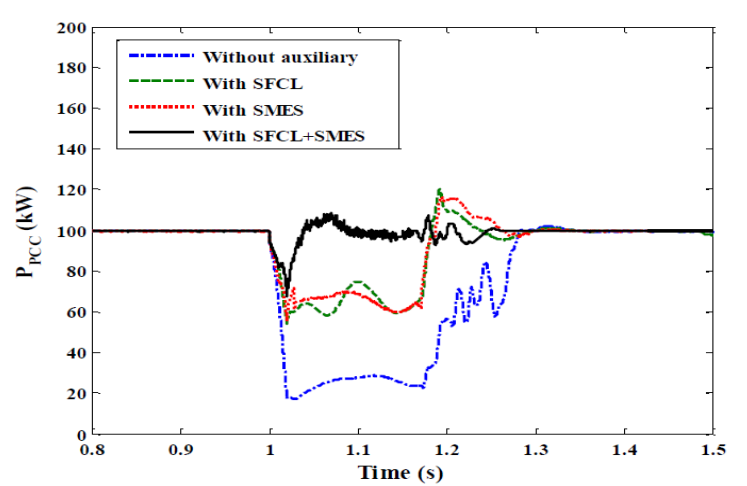

Fig. 1. Characteristic power of PCC during a symmetrical fault [43].

concept has recently been reported that combines a SMES and a battery energy storage (BES) system into a single device. The SMES is ideally suited to handling the short-term instantaneous power while the battery complements this through supplying long-term continuous power thanks to its large energy density and long cyclic life. This complementary SMES-battery hybrid form of ES presents a more techno-economical application of SMES for power grid applications as it reduces the capital cost constraint of the SMES due to a lower capacity requirement. Such a system has also been shown through simulation to be useful in electric buses [30], all electric ships [31] in electric vehicle charging stations [32] and in combined heat and power systems [33].

The interconnected microgrid has received lots of attention for accommodating distributed RES and presents an interesting application of SMES-based systems with various studies indicating improvement in battery lifetime up to $60 \%$ [34], enhanced fault ride through capability as well as frequency control and support during operation mode switching (islanded/grid-connected) [35]. An ES device can provide crucial stability during mode switching by rapidly compensating power differences however it is obvious that there is no single device that can meet both the technical and economical requirements of growing microgrid installations. Thus, incorporating ES in a hybrid form (e.g. battery-SMES) is technically and economically advantageous through the improvements it can bring which in turn, can enhance return value and provide increased incentives for RES integration. It could be anticipated that the initial introduction of SMES systems on a commerical scale could come in supporting microgrids and RES farms such as wind and solar, with initial deployment in hybrid form to benefit from the discussed technical and economical advantages. However, with many reports being solely based on simulation and very little in the way of physical demonstrative projects, implementation is still some way off in comparison to the other technologies. Despite this, there remains intriguing research frontiers with regards to providing suppoort to integration of RES through way of frequency, voltage and power quality improvement.

\section{Other Notable Technologies}

The transformer is another conventional technology where the application of superconductivity can offer additional 
benefits with existing substation sites being uprated to higher power capabilities due to limited siting availability in urban areas. The reduced size and weight of a superconducting transformer, coupled with benign $\mathrm{LN}_{2}$ as opposed to oil as the coolant presents a potentially more viable and significantly safer option, especially in densely built up or indoor sites such as skyscrapers. They are also able to operate at twice their rating (at a sacrifice of efficiency) at no cost to the overall service lifetime and thus can effectively carry the load of two conventional transformers at once in times of emergency [36]. However, successful fabrication and demonstration is sparse across the industry and currently limited to design, simulation and small scale prototype testing [5]. Despite this a 3-phase $630 \mathrm{kVA} / 10.5 / 0.4 \mathrm{kV}$ HTS transformer has been in operation in a live substation in China since 2011 and is to be upgraded to $1250 \mathrm{kVA}$ due to increasing load concerns [37]. Superconducting transformers are arguably one of the most difficult of the superconducting technologies to realise due to the need for very low AC losses and fault current protection and as such are believed to be the furthest from commercialisation with considerable R\&D still required [5].

Another frontier of superconducting technology for the power grid is in superconducting generators that bring improved performance, efficiency and a particularly smaller footprint with most superconducting machines currently incorporating a hybrid design of a superconducting rotor and conventional stator [38]. Wind turbines is the main application for such a technology with the strive for lighter, more compact generators. Common failures of gearboxes considerably reduce the reliability of off-shore wind turbines and as such, there has been a trend towards omitting the gearbox entirely through introducing direct drive technologies [39] and a $10 \mathrm{MW}$ superconducting generator for such an application is being designed employing $\mathrm{MgB}_{2}$ superconducting coils in the rotor providing a weight reduction of $26 \%$ [40]. Although a superconducting wind turbine generator is an interesting solution, it is not currently economically feasible at the ratings of around 5MW of current conventional turbines and instead, the superconducting drive train is expected to become practical at power ratings of $10-15 \mathrm{MW}$ in tandem with an anticipated drop in material and manufacturing costs [39]. It is suggested that market maturity for superconducting generators will not be realised until beyond 2030, with the demonstration stage of such a turbine yet to be realised [5]. Despite this, there is strong evidence of interest on a national scale with the U.S. Department of Energy announcing approximately $\$ 25 \mathrm{~m}$ of funding for 13 projects under the 'Next Generation Electric Machines Program' aimed at advancing technologies for energy-efficient electric motors, $\$ 15 \mathrm{~m}$ of which was consigned specifically to research teams in superconductivity [41].

\section{Combining Superconducting TeChnOlogies}

It is inevitable that with the development of multiple superconducting devices that they will eventually co-exist in the same grid at the same time, operating alongside one another. In China, a $10.5 \mathrm{kV}$ superconducting substation was constructed that incorporates a $1 \mathrm{MJ} / 0.5 \mathrm{MVA}$ SMES active alongside a
1.5kA SFCL, $75 \mathrm{~m} / 1.5 \mathrm{kA}$ cable and a $630 \mathrm{kVA}$ transformer for the purposes of power quality improvement and was the first of its kind to be installed anywhere. Since its inception in early 2011, it has been providing high quality power to three hightech industrial firms [42].

Investigations into the operation of SMES operating in tandem with an SFCL has also presents considerable improvements in FRT capabilities of microgrids and connected RES particularly wind farms where variable wind speeds cause output power fluctuations.

Fig. 1 displays the power at the Point of Common Coupling (PCC) of the microgrid with the main network [43] and demonstrates how a combination of a SFCL and SMES operating together can greatly enhance FRT and power smoothing capabilities of a microgrid with a power drop of only $4 \%$ with both and $75 \%$ without either. This can aid greatly in adhearing to strict grid codes, allowing microgrids and RES to remain connected rather than face disconnection from the grid during faults. Recent studies have also shown that a SFCLSMES combination is successful in improving frequency and voltage control for systems with high wind penetration in particular [44] with successful testing of a 1MVA-1MJ SFCLSMES system implented in a wind farm in the northwest of China demonstrating signifcant improvements in power quality and reliability of wind generation [45].

\section{COMmon CHALlengeS}

Across each of the technologies discussed there remain common hindrances. The High Temperature Superconductivity Roadmap for the Electric Power Sector 2015-2030 [5] discusses several factors limiting the progress of the aforementioned power applications and can be summarised by the table in Fig. 2 indicating that the lack of long term experience, standardised testing procedures and large investment costs are still worse than current technologies The potential however is clearly evident when considering the positive influences the technologies can have on the grid in terms of size, response, and safety to name a few [5].

Perhaps the most publicised hindrance is a required reduction in price of raw HTS material. A feasibility study examining the potential implementation of superconducting cables in distribution networks to solve capacity concerns concluded that a superconducting solution presently would be $75 \%$ more expensive than the conventional. However, if the trend in price of superconducting materials continues to drop

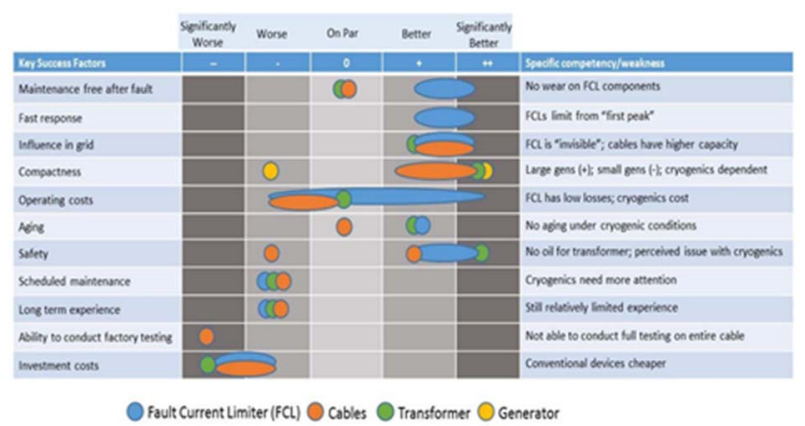

Fig. 2. Key performance factors of superconducting devices measured against their conventional counterparts [5]. 
by approximately $10 \%$ per year, then a $2 \mathrm{~km}$ cable would be comparable to the conventional in approximately 5 years and a $5 \mathrm{~km}$ cable comparable in about 10 years [46]. There is potential that the success and benefits from the European Commission FASTGRID project - which aims to develop improved, more economically feasible superconducting material architecture for SFCLs in HVDC supergrids - could cascade into other applications of superconductivity, with the development of advanced and more economical high temperature superconducting materials [47]. With current medical applications of superconductivity such as Magnetic Resonance Imaging (MRI) primarily based on low temperature superconductors (LTS), the advancement towards HTS based medical devices could provide the increased uptake in HTS materials needed to boost overall production levels that would subsequently drive the price of HTS materials down. There has been considerable research over the last few years on this [48] driven by the commissioning of the Commercialization Promotion of High-temperature Superconductivity Project by the New Energy and Industrial Technology Development Organization (NEDO).

A lack of standardised testing facilities and procedures for HTS components and systems mean there is a lack of data available on reliability and undiminished performance over an extended time period $(30+$ years), with accelerated lifetime testing facilities needed. As of 2017 however, there is a newly established working group (WG D1.69) within CIGRE, "Guidelines for test techniques of HTS systems" that is building on previous work to develop dedicated standards for testing [49]. There is also published work in [50] regarding the effects that integration of superconducting technologies can have on existing protection coordination such as distance protection relays, with studies indicating that such technologies can have a negative impact on the reaction time and effectiveness of existing schemes if not properly compensated for.

A general lack of experience and knowledge of the sector amongst utilities and engineers is also of concern, with many unaware of the benefits such innovative technologies can bring and it is suggested that governmental incentives could be introduced to stimulate interest and installation of innovative technologies such as superconductive power devices as well as an increase in outreach and communications.

\section{CONCLUSIONS}

An overview of the main superconductive technologies applicable to the power grid has been provided with their operation, benefits and applications both in research and demonstration reported. Examples of combinations of superconducting technologies have also been appraised along with a discussion around the common challenges inhibiting the widespread uptake. Superconductive cabling and fault current limiters are believed to be at the forefront of the discussed technologies with regards to technological maturity with the most evidence of in-grid demonstrations. Inclusion of both of these technologies in ENTSO-E and IEA roadmaps and correlation of timescales serves to indicate that these technologies are ahead of other superconducting counterparts.
Technologies such as transformers and generators, albeit report potential benefits, are less advanced in terms of market maturity with the expected timeline beyond 2030 in published roadmaps. It is anticipated that superconductive cabling will continue to make an impact on a distribution level thanks to the progress of demonstration projects, but also with the advancement of DC technologies at a transmission level, has the ability to be incorporated into multi-terminal networks however a successful demonstration of this is yet to be presented as demonstrated cable lengths are limited to $2 \mathrm{~km}$ at present. Application to microgrids is a key active frontier, with discussions presented around the benefits that SFCL and SMES can bring both operating alone or in tandem with each other and presents an area for potential further research and development.

\section{REFERENCES}

[1] UK Energy and Climate Change Committee, "Low Carbon Network Infrastructure: First Report of Session 2016-17," House of Commons, 2016.

[2] K. Qian, Z. Guo, Y. Terao and H. Ohsaki, "Electromagnetic and thermal design of SFCL for DC electric systems using superconducting," in 20th International Conference on Electrical Machines and Systems (ICEMS), Sydney, 2017.

[3] Electric Power Research Institute (EPRI), "Superconducting Power Equipment: Technology Watch 2012,” 2012.

[4] European Network of Transmission System Operators for Electricity (ENTSO-E), "Research, Development and Innovation Roadmap 20172026," 2017.

[5] B. G. Marchionini, Y. Yamada, L. Martini and H. Ohsaki, "HighTemperature Superconductivity: A Roadmap for Electric Power Sector Applications, 2015-2030," IEEE Transactions on Applied Superconductivity, 2017.

[6] M. Stemmle, F. Merschel and M. Noe, "Ampacity Project - Update on World's First Superconducting Cable and Fault Current Limiter Installation in a German City Centre," in CIRED 23rd International Conference on Electricity Distribution, Lyon, 2015.

[7] S. Lee, J.-J. Lee, J. Yoon, Y.-W. Kang and J. Hur, "Impact of 154-kV HTs Cable to Protection Systems of the Power Grid in South Korea," IEEE Transactions on Applied Superconductivity, vol. 26, no. 4, 2016.

[8] Sumitomo Electric, "High Tc Superconducting Cable Project," February 2014. [Online]. Available: http://globalsei.com/super/cable_e/ingridj.html. [Accessed 21 December 2017].

[9] J. X. Jin, R. Islam and A. G. Khan, "Development of HTs Cable-Based Transmission Systems for Renewables," in Renewable Energy and the Environment, Springer, 2018, pp. 71-114.

[10] P. Rodriguez and K. Rouzbehi, "Multi-terminal DC grids: challenges and prospects," Journal of Modern Power Systems and Clean Energy, vol. 5, no. 4, pp. 515-523, 2017.

[11] A. Ballarino et al., "The BEST PATHS Project on MgB2 Superconducting Cable for Very High Power Transmission," IEEE Transactions on Applied Superconductivity, vol. 26, 2016.

[12] X. Pei, A. C. Smith and M. Barnes, "Superconducting Fault Current Limiters for HVDC Systems," Energy Procedia, vol. 80, pp. 47-55, 2015.

[13] Electric Power Research Institute (EPRI), "Superconducting Fault Current Limiters: Technology Watch,” 2009.

[14] Siemens, "Siemens: Superconducting Technology - Fault Current Limiters," $2014 . \quad$ [Online]. Available: https://www.siemens.com/press/pool/de/pressemitteilungen/2014/corp orate/factsheet-supraleiter_e.pdf. [Accessed 24 January 2018].

[15] G. Angeli et al., "Status of Superconducting Fault Current Limiter in Italy: Final Results from the In-Field Testing Activity and Design of 
the 9kV/15.6MVA Device," IEEE Transactions on Applied Superconductivity, vol. 26, 2016.

[16] Nexans, "Birmingham's Power Grid is now Reinforced with Nexans' Superconducting Fault Current Limiters," March 2016. [Online]. Available: $\quad$ https://www.nexans.com/Corporate/2016/1603Nexans_WPD_Birmingham_GB.pdf. [Accessed 2018 January 25].

[17] BINE Information Service, "Stadtwerke Augsburg testing superconducting fault current limiter," 21 March 2016. [Online]. Available: http://www.bine.info/en/topics/news/stadtwerke-augsburgerproben-supraleitenden-strombegrenzer.pdf. [Accessed 2 February 2018].

[18] L. Chen et al., "Comparison of Superconducting Fault Current Limiter and Dynamic Voltage Restorer for LVRT Improvement of High Penetration Microgrid," IEEE Transactions on Applied Superconductivity, vol. 27, no. 4, 2017.

[19] R. Haider et al., "Protection Coordination Using Superconducting Fault Current Limiters in Microgrids," Journal of the Korean Institute of IIIuminating and Electrical Installation Engineers, vol. 31, no. 10, pp. 26-36, 2017.

[20] U. A. Kahn, J.-G. Lee, F. Amir and B.-W. Lee, "A Novel Model of HVDC Hybrid-Type Superconducting Circuit Breaker and Its Performance Analysis for Limiting and Breaking DC Fault Currents," IEEE Transactions on Applied Superconductivity, vol. 25, no. 6, 2015.

[21] S. Liang et al., "Test and Analysis of a Small-Scale Hybrid-Type DC SFCL Prototype," IEEE Transactions on Applied Superconductivity, vol. 28 , no. $4,2018$.

[22] H.-S. Choi et al., "Superconducting DC Circuit Breaker using Arcing Induction". China Patent US20170316894A1, 2 November 2017.

[23] L. Chen, F. Tang and L. Ren, "Comparitive Study of Inductive and Resistive SFCL to Mitigate the DC Fault Current in a VSC-HVDC System Integrated with Wind Power Farms," in Proceedings of 2015 IEEE International Conference on Applied Superconductivity and Electromagnetic Devices, Shanghai, China, 2015.

[24] H.-Y. Lee, M. Asif, K.-H. Park and B.-W. Lee, "Feasible application Study of Several Types of Superconducting Fault Current Limiters in HVDC Grids," IEEE Transactions on Applied Superconductivity, vol. 28, no. 4, 2018.

[25] A. B. Gallo, J. R. Simoes-Moreira, H. K. M. Costa, M. M. Santos and E. Moutinho dos Santos, "Energy storage in the energy transition context: A technology review," Renewable and Sustainable Energy Reviews, vol. 65, pp. 800-822, 2016.

[26] M. Aneke and M. Wang, "Energy storage technologies and real life applications - A state of the art review," Applied Energy, vol. 179, pp. 350-377, 2016.

[27] D. O. Akinyele and R. K. Rayudu, "Review of energy storage technologies for sustainable power networks," Sustainable Energy Technologies and Assessments, vol. 8, pp. 74-91, 2014.

[28] A. Alhamali, M. E. Farrag, G. Bevan and D. M. Hepburn, "Review of Energy Storage Systems in Electric Grid and their Potential in Distribution Networks," in 18th International Middle East Power Systems Conference (MEPCON), Cairo, 2016.

[29] A. Morandi et al., "The DRYSMES4GRID Project: development of a $500 \mathrm{~kJ} / 200 \mathrm{~kW}$ cryogen-free cooled SMES demonstrator based MgB2," IEEE Transactions on Applied Superconductivity, 2018.

[30] J. Li, M. Zhang, Q. Yang, Z. Zhang and W. Yuan, "SMES/Battery Hybrid Energy Storage System for Electric Buses," IEEE Transactions on Applied Superconductivity, vol. 26, no. 4, 2016.

[31] H. Alafnan et al., "Stability Improvement of DC Power Systems in an All-Electric Ship Using Hybrid SMES/Battery," IEEE Transactions on Applied Superconductivity, 2018.

[32] J. Deng, J. Shi, Y. Liu and Y. Tang, "Application of a hybrid energy storage system in the fast charging station of electric vehicles," IET Generation, Transmission, Distribution, vol. 10, no. 4, pp. 1092-1097, 2016.
[33] J. Li et al.,"Analysis of a new design of the hybrid energy storage system used in the residential m-CHP systems," Applied Energy, vol. 187, pp. 169-179, 2017.

[34] J. Li et al., "A novel use of the hybrid energy storage system for primary frequency control in a microgrid," Energy Procedia, vol. 103, pp. 8287, 2016.

[35] Y. Xu et al., "Research on the Application of Superconducting Magnetic Energy Storage in Microgrids for Smoothing Power Fluctuation Caused by Operation Mode Switching," IEEE Transactions on Applied Superconductivity, vol. 28, no. 4, 2018.

[36] V. Upadhye and U. C. Savardekar, "Superconducting Transformers," International Journal of New Innovations in Engineering and Technology, vol. 4, no. 3, pp. 67-73, 2016.

[37] S. Dai, T. Ma, Q. Qiu, Z. Zhu, Y. Teng and L. Hu, "Development of a 1250kVA Superconducting Transformer and its Demonstration at the Superconducting Substation," IEEE Transactions on Applied Superconductivity, vol. 26, no. 1, 2016.

[38] M. Ainslie, M. Izumi and M. Miki, "Recent advances in superconducting rotating machines: an introduction to the 'Focus on Superconducting Rotating Machines'," Superconductor Science and Technology, vol. 29, 2016.

[39] A. B. Abrahamsen, N. Magnusson, B. B. Jensen and M. Runde, "Large superconducting wind turbine generators," Energy Procedia, vol. 24, pp. 60-67, 2012.

[40] I. Marino et al., "Lightweight MgB2 Superconducting 10MW Wind Generator," Superconductor Science and Technology, vol. 29, 2016.

[41] IEA Technology Collaboration Program on High Temperature Superconductivity, "Annual Report 2016," International Energy Association, 2017.

[42] S. Dai et al., "Development and Demonstration of a 1MJ High-Tc SMES," IEEE Transactions on Applied Superconductivity, vol. 22, no. $3,2012$.

[43] L. Chen et al., "Coordinated Control of Superconducting Fault Current Limiter and Superconducting Magnetic Energy Storage for Transient Performance Enhancement of Grid-Connected Photovoltaic Generation System," Energies, vol. 10, no. 1, 2017.

[44] S. M. Said, M. M. Aly and B. Hartmann, "Application of SMES for Voltage Control of Power Systems with High Wind Penetration," in International Conference on Innovative Trends in Computer Engineering (ITCE), Egypt, 2018.

[45] W. Guo et al., "Development of a 1MVA/1MJ Superconducting Fault Current Limiter-magnetic Energy Storage System for LVRT capability enhancement and wind power smoothing," IEEE Transactions on Applied Superconductivity, vol. 28, no. 4, 2018.

[46] W. Yuan, S. Venuturumilli, Z. Zhang, Y. Mavrocostanti and M. Zhang, "Economic Feasibility Study of using High Temperature Superconducting cables in UK's Electrical Distribution Networks," IEEE Transactions on Applied Superconductivity, 2018.

[47] European Commission, "FASTGRID - Cost effective FCL using advanced superconducting tapes for future HVDC grids," 1 January $2017 . \quad$ [Online]. Available: https://cordis.europa.eu/project/rcn/206750_en.html. [Accessed 7 June 2018].

[48] M. Oya et al., "Design and Manufacture of Half-Size 3-T HighTemperature Superconducting Magnet for MRI," IEEE Transactions on Applied Superconductivity, vol. 28, no. 3, 2018.

[49] CIGRE: WG D1.69, "Proposal for the Creation of a New Working Group: Guidelines for test rechniques of High Temperature superconducting (HTS) systems," 2017.

[50] J. Yang, L. Zhang, X. Wang, L. Chen and Y. Chen, "The impact of SFCL and SMES integration on the distance relay," Physica C: Superconductivity and its Applications, vol. 530, pp. 151-159, 2016. 